\title{
Exploring the Business Case Development Process in Inter-Organizational Enterprise System Implementations
}

Silja Eckartz, University of Twente, The Netherlands

Christiaan Katsma, University of Twente, The Netherlands

Maya Daneva, University of Twente, The Netherlands

\begin{abstract}
Creating and negotiating an inter-organizational business case (BC) for multiple-stakeholder enterprise systems is a major challenge. This paper looks closer into the factors that influence the stakeholders 'willingness to share information necessary for the $B C$ development. The authors develop an explanatory framework showing the effect that project constellation has on the development of a shared BC. They identify several factors, such as goal consensus, cultural and semantic similarities and willingness to share information, that mitigate this effect. Subsequently, the authors apply the framework in an inter-organizational case study in which a BC is developed. The findings show that current $B C$ development methods need to be re-stated and complemented by new tools and interventions to support stakeholders in the inter-organizational specific setting. The authors elaborate on the possibilities that group decision support systems might offer to overcome challenges that might be experienced in the $B C$ development process.
\end{abstract}

Keywords: $\quad$ Business Case, Decision Making, EnterpriseSystems, Information Sharing, Inter-Organizational Coordination

\section{INTRODUCTION}

A business case (BC) describes and specifies the costs, benefits and risks for a project. This paper focuses on the process of deploying a $\mathrm{BC}$ for IT projects, such as information system (IS) implementations. Most literature on BC's, cost-benefit analysis and information technol-

DOI: $10.4018 /$ irmj.2012040106 ogy (IT) evaluation discusses projects that are implemented by a single organization. In today's business practice, however, it is often important to address the situation in which multiple actors are involved in a joint project. In the actual deployment of such a project one speaks of the implementation of an inter-organizational system (IOS). A multi organizational business case development (BCD) process is deployed prior to the implementation decision, during 
which the actors evaluate together if it is valuable to invest into the project.

In this paper we analyze the $\mathrm{BCD}$ process for the evaluation of an inter-organizational Enterprise System (ES). We investigate how different project constellations influence the $\mathrm{BCD}$ process especially when multiple actors are involved in the ES implementation project.

Enterprise systems are commercial software packages that enable the seamless integration of information and business processes within and across functional areas in an organization (Kumar \& van Hillegersberg, 2000). Today ES do not only enable the integration and coordination within one organization, but also go one step further and support the connection and management of information and business processes across several organizations. Cash and Konsynski (1985) define an IOS as "an automated information system shared by two or more companies." It enables inter-organizational coordination between profit-and-loss responsible business units, or between independent companies (Bakos, 1991).

In the case of ES this crossing of organizational boundaries not only increases the complexity but also implies substantial differences in semantics, processes, information and goals between the different actors (Daneva \& Wieringa, 2006). The multiple actors engaged in inter-organizational coordination often encounter problems when they need to share information in order to make important joint decisions. In our research, we focus specifically on the process of how multiple actors arrive at a joint decision about whether or not to invest in an inter-organizational ES. More specifically, we will analyze the process of shared business case development (BCD). We do so by first investigating the constellation structure of the project participants and secondly by specifying the properties that affect the BCD process.

Our main research question is: What are the effects of project constellations on the process of developing an inter-organizational business case for IS implementations?

The main contribution of this paper is to extend the body of knowledge on BCD during
IS implementations. Our paper especially addresses the context of IOS, which has been a rather underexposed research area. We contribute to the body of exploratory research on the factors that play a role during the $\mathrm{BCD}$ process. Moreover, we explore coordination properties, such as competition, semantics, culture and willingness to share information, which improves our understanding of how BCD takes place in large shared enterprise systems projects.

\section{THEORETICAL BACKGROUND}

This section summarizes the findings of the current state of the literature as well as of the available empirical studies. For clarification purposes and to position our line of reasoning we start defining what we mean by the concept of "Business Case."

We use the definition of a Business Case as an artifact (possibly a document accompanied by designs or models, etc.) that specifies the main rationale and expected value for the ES-adopting organization. The $\mathrm{BC}$ evaluates the different implementation options, based on the expected costs, benefits and risks of each option during the entire implementation process (Schubert \& William, 2009; Shang \& Seddon, 2002; Ward \& Daniel, 2006). Ideally it contains more than just a financial analysis. The (non-) financial benefits, business alignment, costs and risks, should be complemented with information on the methods and rationale that were used to quantify the benefits and costs (Schmidt, $2003 \mathrm{~b}$ ). The $\mathrm{BC}$ is the result of a BCD process that is deployed between consultants and stakeholders from the ES-adopting organization. The $\mathrm{BCD}$ is an iterative, tool-supported process that relies on stakeholders from different parts of the organization with different business knowledge.

In the field of IS research, scholars take different perspectives upon this relatively young research domain (Klein, Kupsch, \& Scheer, 2004; Schulz \& Orlowska, 2004). Our research builds upon the work of Kishore et al. (2004) that includes coordination theory to exemplify the extra complexity due to the involved actors. We 
Figure 1. Research model

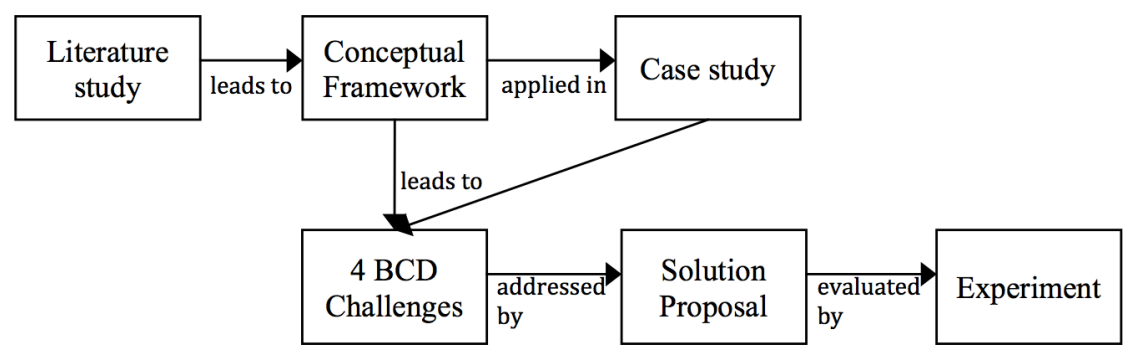

extend their line of reasoning by investigating the impact of the project constellation among the involved actors when the $\mathrm{BC}$ is initiated during the early stages of an inter-organizational ES implementation.

The network of involved actors should deliver diverse inputs to build a $\mathrm{BC}$ for an inter-organizational ES implementation, which complicates the BCD process (Schmidt, 2003b). Although the actors are aware of the need to share information, they might lack a shared understanding of the terminology used, hesitate to release sensitive information or disagree on how costs and benefits are distributed in a network. The involved actors might further have different drivers for participating in an inter-organizational cooperation, such as acquire resources, reduce uncertainty, enhance legitimacy or, in the best case, attaining collective goals (Oliver, 1990). Differences in these drivers among actors can make the $\mathrm{BCD}$ process challenging and complex and consequently this calls for a structured BCD approach.

\section{RESEARCH METHOD}

This paper presents results that are part of an ongoing research project on the implementation of inter-organizational IS and ES in particular. We deployed the following research approach, which is shown in Figure 1.

We first conducted an extensive literature review using the guidelines of Webster and Watson (2002). We included publications covering BCD (Ward \& Daniel, 2006; Ward, Daniel, \& Peppard, 2008), coordination mechanisms (Crowston, 1997; Kishore et al., 2004; Malone \& Crowston, 1994), and interorganizational ES implementation(Davenport, Harris, \& Cantrell, 2004; Ferrario \& Montagna, 2004) in our literature review. This review resulted in relevant findings on the deployment of the BC during an inter-organizational ES implementation. Via induction and clustering (Rowley \& Slack, 2007) we identify four factors that influence the quality of the BCD process. We evaluate these findings from the perspective of project constellations in inter-organizational collaborations and derive our explanatory framework. The objective of our framework is to specify the increased complexity of the $\mathrm{BCD}$ process during the early stages of the interorganizational ES implementation.

Next, we deploy our framework in a case study of the Rotterdam Harbor. Rotterdam Harbor is a large business network in the transportation sector in the Netherlands. Figure 2 shows an overview of how the different actors in the network are linked through contracts as information flow or the physical flow of containers.

We deployed participative observation techniques in workshops within 6 consecutive months. We also conducted interviews, observed negotiation between actors, and, in a few occasions, guided the attempt to develop a shared $\mathrm{BC}$ for the network. We used a diary approach to record incidents. Also, we reflected on the relevant events with different interviewees in informal unstructured interviews. We participated in the following sessions: 
Figure 2. Network structure of the case

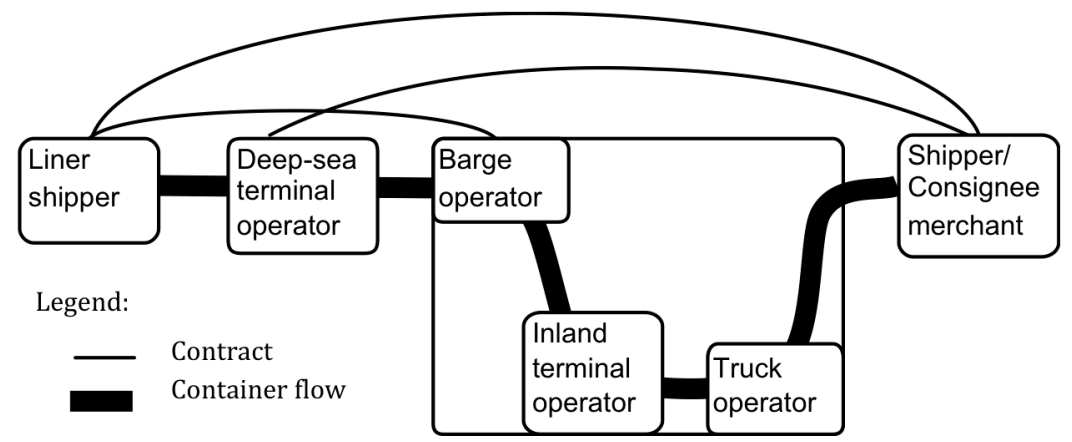

- 6 individual interview meetings with different actors to get an understanding of the situation at hand and of the important concerns of each actor. Unstructured interviews were conducted to collect mostly qualitative data.

- 5 meetings with different stakeholders (several barge operators, the Transportation ministry, and an official from the Harbor of Rotterdam).

- 3 collaborative workshops with most stakeholders present except for the terminal operators.

We coded and clustered the information that was collected by means of our interviews and the other empirical materials using our conceptual framework. Based on the analysis of the case study results we are able to identify four challenges that companies are expected to encounter during the BCD process.

We address these challenges in a first solution proposal that we validate with the help of two mirrored experiments. We deploy master students in Business and IT as actors in these experiments. The first experimental setting covers a face-to-face traditional meeting and takes place in a room with a stretched oval shaped table. All four participants were facing each other and had sight on the projected steps including the contributing information from the actors that is processed by a moderator.

The second experiment consisted of a web-based group decision support system
(GDSS) meeting and took place in a room with tables placed in a half circular shape. The four participants are facing a TV-screen with an overview of the agenda and the current agenda item processing. All participants were seated behind a laptop with access to the web-based GDSS named Spilter (http://www.spilter.nl).

The participants did not receive any upfront training; they were only given a short instruction on how to $\log$ in and on how the experiment would proceed.

The real experiments are preceded twice by a pilot with fellow researchers taking the roles of the different actors. This way the case and script for the experiment are validated and finalized. In total four rounds of experiments are deployed (two per setting). For each round four different actors are involved. A questionnaire was used to compare the mirrored experiments.

\section{Assumptions About the Context in Which Our Framework Will be Valuable}

In this paper we set out to develop a framework that explains how different factors can influence the BCD process. This section elaborates on the assumptions we make in our explanatory framework.

Inter-organizational relations can be classified based on the type of relationship between partners and their coordination structure. A distinction can be made between markets and non-markets (Heide, 1994). Markets are char- 
Figure 3. Different coordination structures and their power relation (adapted from van Alstyne, 1997)

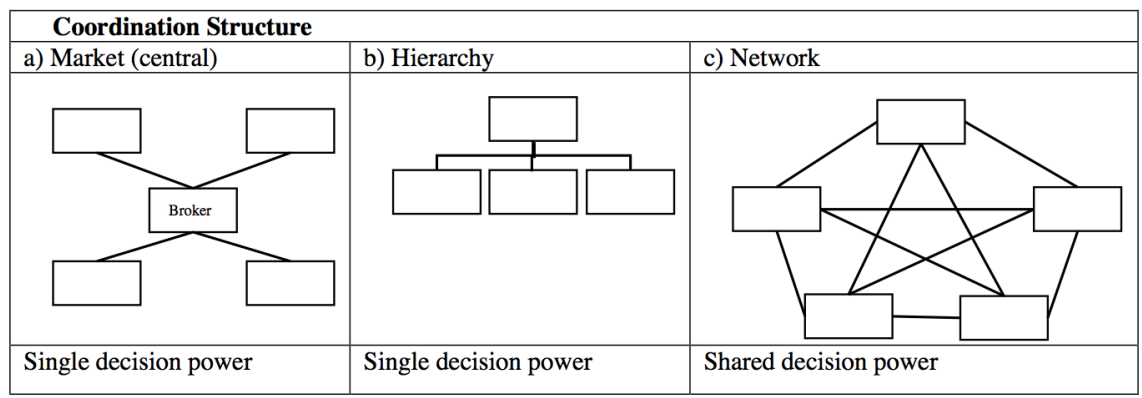

acterized by discrete interactions and limited personal involvement (Powel, 1990), while non-market interactions are usually based on some form of relationship between the partners. The latter can be classified as being either of hierarchical or of a network nature. Figure 3 shows the three structures.

Inter-organizational relations organized in a market structure (Figure 3a), often have a short-term focus and are mainly based on the price mechanisms. Hierarchical partnership structures (Figure 3b), e.g., franchise or outsourcing contracts, rest on unilateral interaction and an authority relationship. The network (Figure 3c) as a coordination structure is characterized by cooperation, collaboration, and the sharing of information (Jaffee, 2001). Thus, it is different from a pure market structure; in fact, it is a hybrid of hierarchy and market based on bilateral, often long-term interaction between partners.

For our explanatory framework we assume that the implementation of inter-organizational ES involves multiple actors that jointly want/ need to agree on a $\mathrm{BC}$. This is in contrast to existing literature which often assumes a single actor taking ownership for the network and therefore exercising power in the decisionmaking-process. Such single decision power can often be found in coordination structures that have a hierarchical or central market structure (Figure 3). Networks (Figure 3c) include a shared decision power of the actors, where all involved actors should agree on the final BC.
Our framework can most likely also explain the situation for markets and hierarchies (Figures $3 \mathrm{a}$ and $3 \mathrm{~b}$ ) with single decision power, but they are often less complex and therefore do not necessarily need a framework to explain the situation.

Secondly, we assume that the BC is developed for rather complex IS implementations, such as ES or multi agent systems. IS with rather obvious cost and benefits for all stakeholders at the early stage of the project are not considered for our research objective.

\section{AN EXPLANATORY FRAMEWORK FOR BUSINESS CASE DEVELOPMENT IN AN INTER-ORGANIZATIONAL SETTING}

In earlier research we presented an exploratory framework that investigates the impact of coordination structure and project scope on six different coordination properties (Eckartz, Katsma, \& Daneva, 2010).

In this paper we focus on one application of our previous framework, namely the situation in which a $\mathrm{BC}$ is developed for an inter-organizational ES in a network setting with a shared power distribution amongst the actors to make decisions. Our main line of reasoning is depicted in Figure 4, from left to right. In an interorganizational setting we distinguish different project constellations (box (1) in Figure 4). 
Figure 4. Explanatory framework: Impact of project constellation on the BCD process

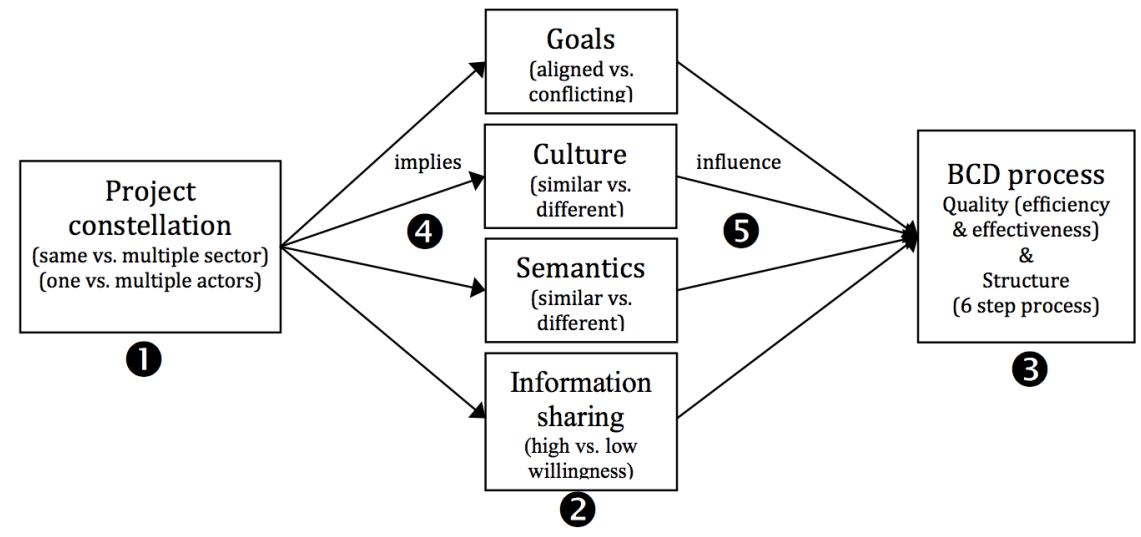

These project constellations influence (arrow (4) the quality of the BCD process ( 3 ) significantly, via four mediating factors (box 2 and arrow $(5$ ).

In the following sections we first explain the three boxes and their relationships respectively. We then conclude the section with a discussion on how specific project constellations result in challenges for the $\mathrm{BCD}$ process.

\section{Project Constellation}

For the purpose of this paper, an actor is defined as a decision-making entity. When referring to a single actor, we mean one organization that may consist of several individuals but each single actor does not have the mere decision making power over the other actors. In this research, we exclusively focus on projects with multiple actors, because we analyze the context of interorganizational systems.

Before the start of a joint project, every single actor is involved in his/her own business network with multiple other actors, such as suppliers, customers or competitors. Several drivers can initiate a joint project like new legislation, innovations or the insight in a shared business opportunity between a set of actors.

The connection between firms in the network is referred to as role linkage by Hong (2002). Hong makes a distinction between horizontal and vertical linkage of the involved actors in an IOS. Horizontal linkage describes the interconnection of firms in the same sector (Figure 5, column a), whereas vertical linkage describes the cooperation between organizations in different sectors, as it is the case in a typical value chain (column b).

Based on Hong's conceptualization of role linkages we discern three different patterns how multiple actors can be involved in such a project ( $a, b$ and $c$, Figure 5). We define the pattern of the number of partners -and sectors as the project constellation. The project complexity increases from column (a) to (c). In short these project constellations can be explained as follows:

Pattern a) A project can be established by actors from one single sector. The implementation of the Dutch payment system IDEAL by several banks is an example of this project constellation (Figure 5, column a).

Pattern b) Single actors from different sectors may be involved in a project, but in pattern $b$ we distinguish exclusively one actor from each sector. Nowadays, these single supplier "pipes" are more exotic as vendors try to balance their risk by multiple supplier partnerships. Still there are substantial situations where component or partner uniqueness often dictates single supplier relationships (Figure 5, column b). 
Figure 5. Different forms of scope of the collaboration

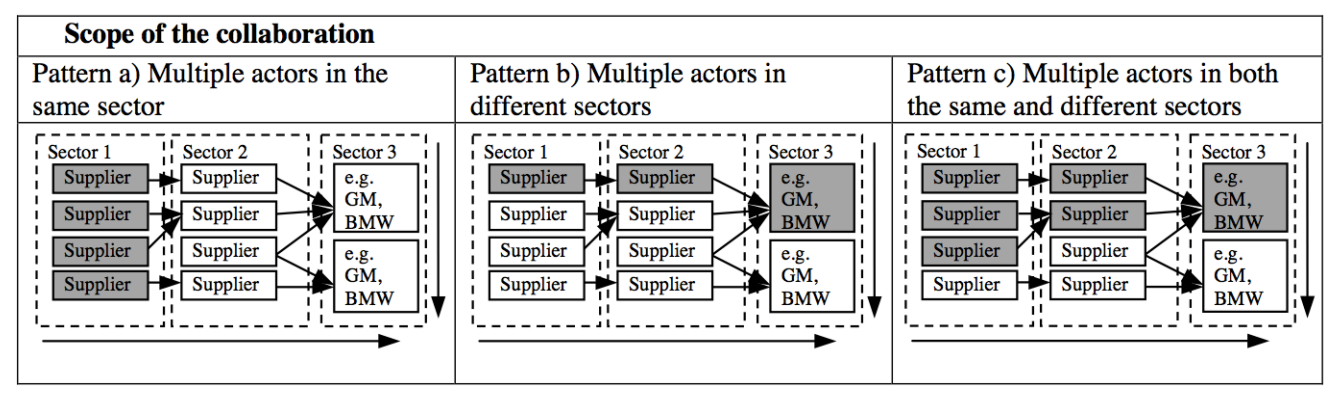

Pattern c)A set of multiple actors from different sectors are involved in the project. There are plenty of examples for this project constellation, e.g., in the automotive industry, where multiple suppliers from different sectors are connected through an IOS with the car manufacturer. (We depicted one possible pattern in Figure 5, column c.)

\section{Four Factors Influencing the Inter-Organizational BCD Process}

Based on our literature review (Daneva \& Wieringa, 2006; Park, 1996; Xu \& Beamon, 2006) and own case research we identify several important factors (Figure 4, box 2 ) that influence the BCD process when evaluating the investment in an IOS. We follow a similar clustering and classification approach like
Rowley and Slack (2007) and derive four main factors that influence the BCD process (Table $1)$. We phrase the factors as discrepancy variables.

\section{Consensus of Goals}

This factor implies that if the individual goals of the actors are conflicting it is more difficult to get an agreement on the $\mathrm{BC}$ than if the goals of the separate actors are aligned. The goals of the individual actors are specific for a project as they describe the individual objectives that each actor wants to achieve with the project. Individual goals can be similar or different among the actors and can even change throughout the project. If the goals are different, they can either be aligned or they could be in conflict with the goals of the other actors. Consensus between

Table 1. Four factors influencing the BCD process

\begin{tabular}{|l|l|l|}
\hline Factor & Explanation & Sources \\
\hline Consensus of goals & $\begin{array}{l}\text { Alignment of the individual goals of each actor } \\
\text { for the project. (We distinguish between aligned } \\
\text { versus conflicting goals) }\end{array}$ & $\begin{array}{l}\text { (Daneva \& Wieringa, 2006; Davenport, } \\
\text { 1998, 2000; Provan \& Kenis, 2007) }\end{array}$ \\
\hline Cultural similarities & $\begin{array}{l}\text { Similarities in the way the actors interact with } \\
\text { each other (we distinguish between similar versus } \\
\text { different organizational cultures) }\end{array}$ & $\begin{array}{l}\text { (Hofstede, 1983; Kendra \& Taplin, 2004; } \\
\text { Schein, 2004) }\end{array}$ \\
\hline $\begin{array}{l}\text { Semantic } \\
\text { similarities }\end{array}$ & $\begin{array}{l}\text { Similarities in the use of language and knowledge } \\
\text { exchange between the actors (we distinguish be- } \\
\text { tween similar versus different semantics) }\end{array}$ & $\begin{array}{l}\text { (Daneva \& Wieringa, 2006; Davenport, } \\
\text { 2000) }\end{array}$ \\
\hline $\begin{array}{l}\text { Willingness to share } \\
\text { information }\end{array}$ & $\begin{array}{l}\text { Willingness to share sensitive information with the } \\
\text { other actors in the project (we distinguish between } \\
\text { high versus low willingness to share information) }\end{array}$ & (Park, 1996; Thorelli, 1986) \\
\hline
\end{tabular}


Table 2. Quality criteria for the BCD process

\begin{tabular}{|l|l|}
\hline Quality criteria & BCD process specification \\
\hline Efficiency of the BCD process & $\begin{array}{l}\text { Completion of the BC within the projected amount of time, against the } \\
\text { proposed resources }\end{array}$ \\
\hline Effectiveness of the BCD process & $\begin{array}{l}\text { Realization of a qualitative BC that includes the proposed scope, benefits, } \\
\text { cost, risks for the several involved actors. Resolution of possible conflicts } \\
\text { between actors during the process }\end{array}$ \\
\hline
\end{tabular}

the several actors on the majority of the major goals is required for a successful $\mathrm{BCD}$ process.

Consensus is typically a project-related factor as it includes the project goals and the different perspectives of each single actor. The other three factors, semantics, culture and willingness to share information, are typical characteristics that are independent of the project. These three characteristics are typical actor-dependent characteristics.

\section{Cultural Similarities}

This factor states that efficient collaboration requires the awareness of different cultural backgrounds (Hofstede, 1983) and if necessary the application of interventions to create an open and effective group culture (Kendra \& Taplin, 2004). Each of the involved actors has its own organizational culture (Schein, 2004) and the differences between these cultures influence the $\mathrm{BCD}$ process. It is expected that during the $\mathrm{BCD}$ process sub cultures will evolve in which the collection of actors shape their temporary group culture.

\section{Semantic Similarities}

This factor refers to the meanings that actors attach to the information entities and each actors' existing body of knowledge that they would share by using a common IOS. The actors in the network might for example speak a different language or use different terminology. Consequently this could have an impact on the progress or speed of the BCD process and eventually on how agreement on the terms used in the $\mathrm{BC}$ is accomplished (Daneva \& Wieringa, 2006; Davenport, 2000).

\section{Willingness to Share Information}

Information sharing determines the willingness of actors to share their sensitive cost-and benefit information, and their ability to actually supply financial numbers for the BC. It is closely linked to competition between the actors and one of the most important aspects in the BCD process (Thorelli, 1986). Information sharing is easier when actors speak the same language and is more difficult in case of competing actors (Park, 1996).

\section{The BCD Process Specified: Quality Criteria and Structure}

We now analyze the nature of the $\mathrm{BCD}$ process based on quality criteria and structure. To specify the BCD process (Figure 4, box (3) we use common quality criteria. We derive them from project management (like scope, time and resources) and software development processes (Fenton \& Pfleeger, 1996; McCall, Richards, \& Walters, 1977) (efficiency, quality of the output). We specify the quality criteria for the BCD process based on efficiency and effectiveness in Table 2.

Several authors have derived different stepwise approaches to define the BCD process (Remenyi, 1999; Schmidt, 2003a). One of the most commonly used set of guidelines are the ones by Ward et al. (2008), who propose the following six steps for BCD:

1. Define business drivers and investment objectives.

2. Identify benefits measures and objectives.

3. Structure the benefits. 
Table 3. Value of factors in different project constellations (Figure 4 arrow

\begin{tabular}{|l|l|l|l|l|}
\hline Project Constellation & Goals & $\begin{array}{l}\text { Culture (similar } \\
\text { vs. different) }\end{array}$ & $\begin{array}{l}\text { Semantics(similar } \\
\text { vs. different) }\end{array}$ & $\begin{array}{l}\text { Willingness to } \\
\text { share } \\
\text { information } \\
\text { (low vs. high) }\end{array}$ \\
\hline (aligned vs. conflicting) & $\begin{array}{l}\text { Culture (similar } \\
\text { vs. different) }\end{array}$ & Semantics & similar & low \\
\hline (similar vs. different) & $\begin{array}{l}\text { Willingness to } \\
\text { share information }\end{array}$ & different & different & high \\
\hline $\begin{array}{l}\text { (low vs. high) } \\
\text { Pattern a) multiple actors in } \\
\text { the same sector }\end{array}$ & conflicting & different & different & low \\
\hline $\begin{array}{l}\text { Pattern b) multiple actors in } \\
\text { different sectors (exclusive- } \\
\text { ly one actor per sector) }\end{array}$ & conflicting & similar & similar & low \\
\hline $\begin{array}{l}\text { Pattern c) multiple actors in } \\
\text { the same as well as different } \\
\text { sectors }\end{array}$ & conflicting & different & different & high \\
\hline
\end{tabular}

4. Identify organizational changes to achieve benefits.

5. Determine the explicit value of each benefit.

6. Identify costs and risks.

In the next section we will further elaborate on how the $\mathrm{BCD}$ process quality attributes and steps are impacted by the different factors that we specified in the prior sections.

\section{Influence of Project Constellation on the BCD Process}

Based on literature and experience we referred to earlier, we conclude that possible impacts of the factors on the $\mathrm{BCD}$ process can be traced back to different project constellations (Figure 4, arrows (4). For each project constellation pattern we will now specify which of the factors, identified in Table 1, are likely to positively or negatively influence the BCD process. Table 3 depicts the relationship between the three project constellations (specified in Figure 5) and the four mediating factors that influence the BCD process (specified in Table 1).

Table 3 displays the extreme instances of these four factors for each pattern. We focus on situations in which the factors negatively influ- ence the $\mathrm{BCD}$ process as project managers perceive these as being the most critical. When the influence of the factor on the BCD process is likely to be negative it is shaded in grey. We conclude that the following factors are critical: i) conflicting goals, ii) different cultures, iii) different semantics, and iv) low willingness to share information.

The second part of our analysis covers the impact of these "shaded critical factors" on the BCD process (Figure 4, arrows $(5$ ), and displays them in Table 4 . We specify if the effect applies to the whole $\mathrm{BCD}$ process, or to a specific step only.

Beside the impact on the $\mathrm{BCD}$ process steps we expect all four critical factors mentioned in Table 4 to also have a negative effect on the two BCD process quality criteria: efficiency and effectiveness. We will explain our line of reasoning per pattern below, using Tables 3 and 4.

\section{Pattern a}

Pattern $a$, includes all actors from the same sector, and we assume they often have similar motives to participate in a project and are therefore more likely to have similar, non-conflicting 
Table 4. Impact of factors on the BCD process steps (Figure 4 arrow (5)

\begin{tabular}{|l|l|}
\hline Critical Factors & Negative impact on BCD Process Steps \\
\hline Conflicting goals & whole process \\
\hline Different culture & whole process \\
\hline Different semantics & step 3 \\
\hline Low willingness to share information & step 1,2,6 \\
\hline
\end{tabular}

goals (goals=aligned). We further assume that actors in one sector use similar language (semantics $=$ similar) and show comparable behaviors in how they interact with the other actors (culture $=$ similar). However, as actors in the same sector are often competitors we assume that their willingness to share (sensitive) information with other actors is low (willingness= low). Low willingness to share information is expected to hinder the $\mathrm{BCD}$ process as it impedes the collection of important financial information for the $\mathrm{BC}$ from the actors in the project. It will especially impact those steps of the BCD process that rely on the collection of a rather large amount of information, such as it is the case in step 1,2 and 6, where the drivers, objectives, benefit measures, costs and risks for a project are identified. If insufficient information is collected the BCD process will be less efficient and will require more time to be finished.

Group techniques, such as GDSS, might help in overcoming the low willingness to share information, e.g., by supporting anonymous information sharing.

\section{Pattern b}

In pattern $b$, all actors are from different sectors and the problem of low willingness to share information is hardly present as there is, often, no significant competition between the different actors. However, the fact that the actors are from different sectors likely implies that the actors "speak a different language" and are more likely to have different organizational cultures when interacting with each other. Whether or not the semantics of the different actors are similar will determine if the actors can easily obtain a shared understanding of the benefits and consequences of a project. This, in turn, is expected to affect step 3 of the BCD process. In this step multiple actors need to structure the benefits, and thus need to really understand what is meant by the different benefits identified by the multiple actors. Differences in semantics and culture will decrease the efficiency and effectiveness of the BCD process. Further, actors from different sectors are assumed to have different interests in the project, which is reflected in their individual goals. Whether the goals are aligned or conflicting will impact the efficiency of all steps in the BCD process. It further impacts the likelihood of conflict between the actors.

GDSS extended with group culture interventions, might offer possibilities to overcome some of these challenges.

\section{Pattern c}

The challenges identified in type $a$ and $b$ are amplified if the actors in a network are from both the same as well as different sectors. In this situation it is likely that the actors have different goals, semantics and culture. A set of actors also is less likely to share information with the other actors in the network, because competition between them plays a role. Different mitigation strategies should be combined in order to come to a successful BC in this latter situation.

Concluding our analysis we see that the three patterns have different influences on the $\mathrm{BCD}$ process. We now can cluster these 
Table 5. Challenges present in different project constellations and solution proposal

\begin{tabular}{|l|l|l|l|}
\hline Project Constellation & Impact on Factors & Challenge & Solution proposals \\
\hline $\begin{array}{l}\text { Pattern a) multiple actors in } \\
\text { the same sector }\end{array}$ & $\begin{array}{l}\text { Low willingness to share } \\
\text { information }\end{array}$ & Challenge 4 & $\begin{array}{l}\text { Group techniques, such as GDSS, } \\
\text { that allow for anonymous infor- } \\
\text { mation sharing }\end{array}$ \\
\hline $\begin{array}{l}\text { Pattern b) multiple actors in } \\
\text { different sectors (exclusively } \\
\text { one actor per sector) }\end{array}$ & $\begin{array}{l}\text { Conflicting goals } \\
\text { Different culture } \\
\text { Different semantics }\end{array}$ & $\begin{array}{l}\text { Challenge 1 } \\
\text { Challenge 2 } \\
\text { Challenge 3 }\end{array}$ & $\begin{array}{l}\text { Group techniques, such as GDSS, } \\
\text { extended with group culture } \\
\text { interventions }\end{array}$ \\
\hline $\begin{array}{l}\text { Pattern c) multiple actors in } \\
\text { the same as well as different } \\
\text { sectors }\end{array}$ & $\begin{array}{l}\text { Conflicting goals } \\
\text { Different culture } \\
\text { Different semantics } \\
\text { Low willingness to share } \\
\text { information }\end{array}$ & $\begin{array}{l}\text { Challenge 1 } \\
\text { Challenge 2 } \\
\text { Challenge 3 } \\
\text { Challenge 4 }\end{array}$ & $\begin{array}{l}\text { Group techniques, such as GDSS, } \\
\text { intended with group culture } \\
\text { anonymous information sharing }\end{array}$ \\
\hline
\end{tabular}

influences into what we call challenges for the $\mathrm{BCD}$ process.

- Challenge 1: Resolve conflicting goals and objectives of multiple actors in a shared BC.

- Challenge 2: Overcome barriers that hinder the BCD and agreement process due to differences in organizational culture between the actors.

- Challenge 3: Overcome barriers that hinder the $\mathrm{BCD}$ and agreement process due to differences in semantics between the actors.

- Challenge 4: Find ways to increase the willingness of the actors to share (sensitive) information with other actors and to quantify (put numbers in) the $\mathrm{BC}$.

Our aim of this cluster exercise is to propose intervention mechanisms that are specifically applicable in each of the three patterns. Table 5 shows the four grouped challenges for each of the three patterns. It shows that projects with multiple actors from the same sector (type a) are expected to have the least challenges to overcome when developing a $\mathrm{BC}$. Whereas project constellations with pattern $c$ are confronted with the most challenging project situation with respect to the BCD process. Table 5 further provides a first solution proposal for each project constellation that is based on which of the four challenges is present in each pattern.

\section{APPLICATION OF THE FRAMEWORK IN A CASE STUDY}

In order to analyze if our framework helps to explain the BCD process in a real life project we applied it in a case study setting that involves multiple actors in a network that are engaged in the process of making a joint decision on an IT investment. More specifically, barge operators (BO), terminal operators (TO) and the harbor authority discussed how a multi-agent system could support logistic planning in the harbor of Rotterdam. Our case study was part of a bigger research effort: Transumo (www.transumo.nl). A platform, started in 2004, where 150 parties from industry, government and academia jointly develop knowledge about sustainable mobility. We were involved in a business case work package of a larger project (Douma, Schuur, \& Jagerman, 2010) that aimed at the design and implementation of a multi-agent system for the port of Rotterdam. Our role in this case study was inquisitive as well as advisory. In total, three researchers were involved alternately during our 7-month long participation in the case study. We took both expert-based as well as participantobservation-based approaches and conducted various unstructured qualitative interviews to gather information about the $\mathrm{BC}$ development process. While collecting and analyzing the observations and the interviews, we focused on 
Figure 6. Network coordination structure of the case study setting

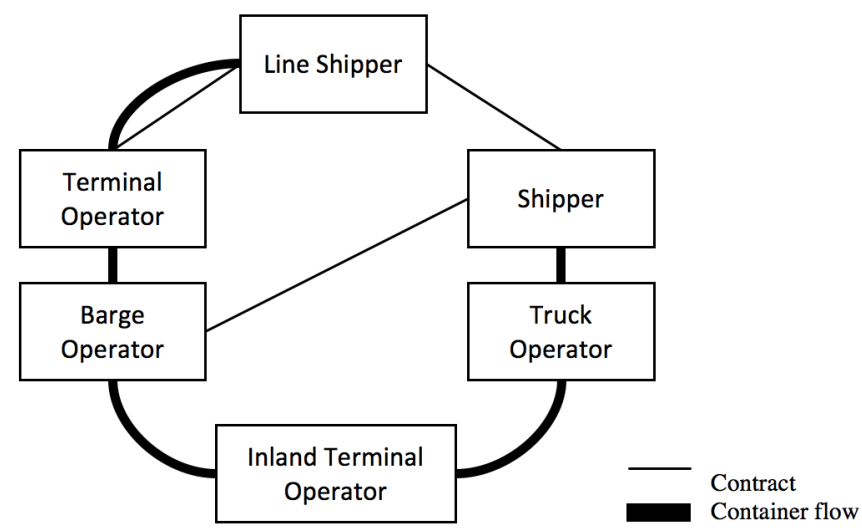

findings related to the $\mathrm{BC}$ development process as well as its outcomes.

In order to assist the actors in evaluating the different implementation options, we supported and helped the participants in workshop settings to develop individual BCs. The idea was to use these individual $\mathrm{BCs}$ as a basis for arriving at a shared BC. Below we explain the impact of project constellation on the shared $\mathrm{BCD}$ process by applying our exploratory framework to the case situation.

\section{Case Background}

The coordination structure shown in Figure 6 presents the relations between the actors in our case setting. Barges are used to transport containers from the port of Rotterdam to the hinterland and vice versa. Whenever a barge visits the port, it has to call on several terminals to load and unload containers. To guarantee short sojourn times in the port, the barge operator schedules convenient arrival times at the concerning terminals. The terminal operators on the other hand want to operate efficiently and have to decide when a barge can be processed, taking into account all kinds of restrictions, e.g., specific times at which containers need to be at the terminal. Based on our knowledge about the project we conclude that the actors in the harbor are organized in a network structure with shared decision power. This implies that the actors in the network need to get to an agreement when making decisions. In this case they need to come to an agreement about their shared BC.

The project constellation is described for the case at hand in Figure 7. One can see that the network involves both actors from the same sector, e.g., several BO's and several TO's, and actors from different sectors (option c in Figure 5). These business characteristics complicate the project scope, e.g., parties want to stay autonomous, have no contractual relationships, and are reluctant to share information that possibly undermine their competitive position.

Douma (2008) shows that an integrated ES, enhanced by multi agent algorithms and controls, can support the alignment of barge rotations and terminal quay capacity, taking into account the business characteristics. We were involved in the $\mathrm{BCD}$ process to evaluate if an investment into such an integrated ES would be profitable.

\section{Application of Conceptual Framework to Case Study}

The application of $\mathrm{BC}$ guidelines developed earlier, turned out to be hardly possible, as participating actors did not share sensitive cost and benefit information. In order to better understand the challenges present in the project at hand we applied our conceptual framework 
Figure 7. Project constellation

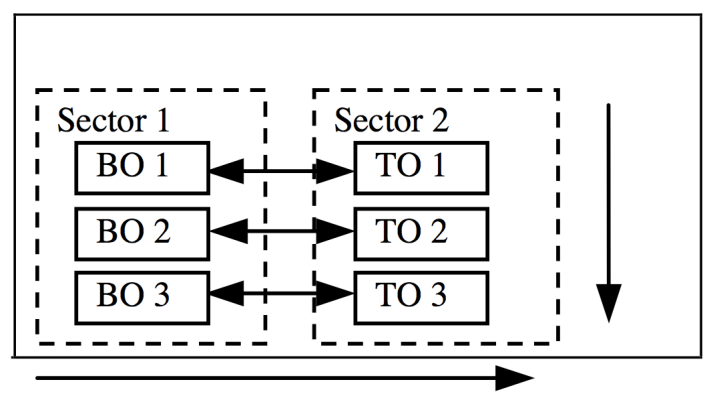

introduced in Figure 4 to our case. The results are shown in Figure 8 and explained afterwards.

Starting from the left, one can see that the harbor case involves several actors from both the same as well as different sectors, as it is illustrated in Figure 7. The actors interact with each other in a network coordination structure, as it is shown in Figure 6. Following from the network structure, the $\mathrm{BCD}$ process results in a shared $\mathrm{BC}$, which is developed and decided on jointly by all actors in the network as they have shared decision power.

The proposition that actors from the same sector experience increased competition compared to actors from different sectors is supported by our case study where we observed high competition especially between the different BOs. This directly impacts the willingness to share sensitive cost and benefit information, which is needed for a shared BC. We found it particularly hard to quantify the expected benefits and costs mentioned vaguely by the different actors. However, without concrete numbers it is very difficult to arrive at a trusted $\mathrm{BC}$, no matter which guidelines one uses. The willingness to share information was further negatively impacted as actors from different sectors did not speak the same language and had different mental models (semantics). This rendered the discussions ineffective as actors had to spend much time on clarifying the meanings of the different terms used in the BC to describe the costs and benefits, e.g., actors had different understandings of what it means to achieve cost reduction or an improvement in planning.

Analyzing the goals of the actors from different sectors in our case study, we found that they were conflicting. The main goal of the BOs is to keep sojourn times short in

Figure 8. Conceptual framework as applied to the case study

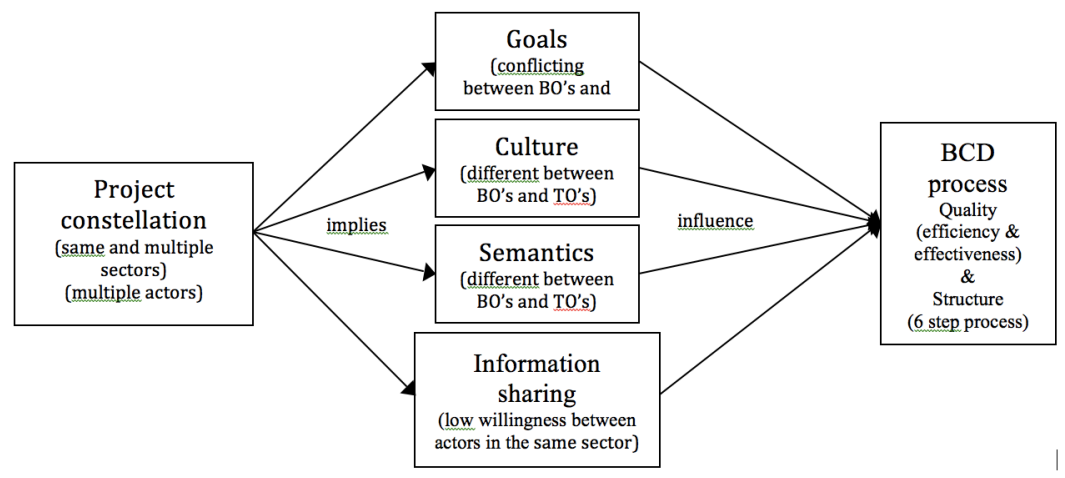

Copyright $($ C 2012, IGI Global. Copying or distributing in print or electronic forms without written permission of IGI Global is prohibited. 
the port and thus waiting times short at the terminal. However, the main goal of the TOs is to have long waiting lines in front of their terminals, so that they always have work for their employees to do. As Figure 6 indicates, in the current situation, there is no contractual relationship between the BOs and TOs; so, no fines will be paid when barges arrive too late at the terminal or when terminals do not handle barges in the agreed time slot. This makes it very difficult to get agreement between BOs and TOs on how a solution could look like. It also makes the $\mathrm{BCD}$ very challenging, as the actors did not agree on the costs and benefits. The TOs actually did not recognize the problem as urgent, as they currently achieve their goal of having long waiting lines, and therefore also had no incentives in investing into an improved planning system.

\section{SOLUTION PROPOSAL}

Our discussion above shows that based on the project constellation, the four factors identified can either support or hinder the BCD process. This solution proposal explicitly focuses on those project constellations that hinder the development of a $\mathrm{BC}$ and, in turn, on how to overcome the challenges experienced in such situations.

Amethod to structure the process is necessary, whenever there are no fixed rules or procedures to deal with the opposing preferences of multiple actors in the process of developing a shared BC (Thompson, 1990). The existing literature identifies four main ways of dealing with opposing preferences: negotiation, mediation, struggle and arbitration (Carnevale \& Pruitt, 1992). Negotiation and mediation have been deemed the most successful as they are less costly and friendlier than struggle. They further make it easier to find an acceptable solution for all actors.

Empirical research has shown that Group Decision Support Systems (GDSS) fit well for highly complex problems with a lack of structure (DeSanctis \& Gallupe, 1987) and can improve decision quality and time efficiency in negotiation processes (DeSanctis, Poole, \& Zigurs, 2008). "A GDSS is a computer-based technology designed to help committees, project teams, and other small groups with activities such as problem identification and analysis, decision making, planning, creativity, conflict management, negotiation, and meeting management" (DeSanctis et al., 2008).

Based on these results, we analyze if and how GDSS can offer ways to overcome some of the challenges that actors may encounter during the inter-organizational $\mathrm{BCD}$ process. We conducted two mirrored experiments twice to compare traditional face-to-face with web-based GDSS sessions when developing a shared BC.

Two experiments were set-up as traditional face-to-face meetings and two were using webbased GDSS to support the BCD process. In all four experiments, a six-step approach, adapted from (Ward et al., 2008), was used to structure the process of $\mathrm{BCD}$. The task of the experiment participants was to develop and agree on a $\mathrm{BC}$ for the implementation of an e-learning environment. This is a case that students can easily relate to. We apply our project constellation patterns to the case, and classify it as pattern b (Figure 5). The actors have conflicting goals and partly a different cultural and semantic background. They are not competitors so that their willingness to share information was normal to high.

Comparing the $\mathrm{BCD}$ process in the two settings we observe the following four advantages of web-based GDSS over face-to-face GDSS:

- Functionality to give anonymous answers.

- Possibility forasynchronouscommunication.

- Prevention of unfunded discussions.

- Focus on content.

We also observe a disadvantage, as the GDSS is not able to completely support all steps of the BCD process. This is due to the fact that the deployed GDSS is not specifically designed for the BCD.

The functionality of the web-based GDSS to give anonymous answers gives all participants in a meeting the chance to freely give their 
opinion and participate in the BCD process. It further allows the actors to anonymously share (sensitive) information that they would not share with the other actors if it could be traced back to them. Our experiments show that anonymous information sharing increases the perceived user satisfaction with the decision quality and also decreases the time to set up the BC.

The web-based GDSS enables asynchronous communication, which allows multiple actors to enter their answers at the same time into the system without influencing the answers of the other participants (Lam \& Schaubroeck, 2000). The system further supports the information gathering process by lowering the barrier of the participants for giving an opinion.

The possibilities for clustering and prioritizing of costs and benefits (arguments), provided by the web-based GDSS prevents unfunded discussions, as participants are required to be consistent in their argumentation. This simplifies the process of opinion forming and prevents misinterpretation of arguments. It further shortens the BCD process, as the discussion is more structured and reduced to well-funded arguing.

We further observed that the strict structure of the web-based system kept the participants away from personal attacks and other distractions that might be present in a face-to-face meeting. Focusing on the BCD process is expected to decrease the time to come to a decision, improve the group communication and improve the satisfaction of the participants with the final decision and the process as a whole.

\section{CONCLUSION}

This paper presents the result of a study that investigated the effects of project constellation on the development of a shared BC for ES implementations in inter-organizational settings. Unlike previously published results, we assume that the power to make decisions is shared by several actors in a network.

Our first contribution is an explanatory framework that makes the ways in which dif- ferent project constellations help or impede the $\mathrm{BCD}$ process explicit. This relation is mitigated by four factors: goal consensus, cultural similarities, semantic similarities and willingness to share information. Our framework fills a gap in the current ES literature, which lacks comprehensive studies on $\mathrm{BC}$ decision-making in interorganizational settings. Based on our framework we identify four challenges that organizations might encounter when developing the $\mathrm{BC}$. The existence of these challenges depends on the project constellation. The identification of these four challenges is our second contribution of this paper. As a third contribution we are elaborating on the possibilities web-based GDSS offer to mitigate the four challenges identified through our framework.

The first application of our framework in a case study demonstrated that it made sense and was useful. To gain a deeper insight into the effects of coordination structure on the $\mathrm{BCD}$ process, we intend to use the framework as structure for further empirical investigations about shared BC in ES implementations.

Our framework has some implications for both practitioners and researchers. For practicing project managers, we think that if they are aware of the four challenges and the inter-organizational mechanisms in place, they could devise strategies to mitigate their impact on the BCD process. For example, a project manager may well consider using a GDSS as a key component in his/her strategy to establish collaboration and consensus-building spirit and make the workplace more comfortable for participants to share information and build upon each other insights and inputs into the BCD process. A project manager can also use the framework to understand his/her own settings in terms of possibilities and restrictions that these settings mean to everyone in the project. If a project manager needs a solution strategy for one or more common problems (e.g., goal misalignment, cultural discrepancies, semantic dissimilarities and low level of willingness to share information), he or she would better know what strategy is realistic for her context and what actions would be doomed to fail. 
For researchers, our framework could serve as an explanation vehicle that can be used in future case study research. As indicated earlier, we are interested in accumulating experiences, which could evaluate the relationships between the concepts in our framework. Only then, we can consider our framework to be more complete. We, therefore, welcome other researchers engaged in research studies similar to ours to consider including our model in their research so that we can use their experiences to confirm or disconfirm the relationship among the concepts in our framework.

\section{REFERENCES}

Bakos, J. (1991). A strategic analysis of electronic marketplaces. Management Information Systems Quarterly, 295-310. doi:10.2307/249641

Carnevale, P., \& Pruitt, D. (1992). Negotiation and mediation. Annual Review of Psychology, 43(1), 531582. doi:10.1146/annurev.ps.43.020192.002531

Cash, J. I. Jr, \& Konsynski, B. R. (1985). IS redraws competitive boundaries. Harvard Business Review, 63(2), 134-142.

Crowston, K.(1997). Acoordination theory approach to organizational process design. Organization Science, 8(2), 157-175. doi:10.1287/orsc.8.2.157

Daneva, M., \& Wieringa, R. J. (2006). Arequirements engineering framework for cross-organizational ERP systems. Requirements Engineering, 11(3), 194-204. doi:10.1007/s00766-006-0034-9

Davenport, T. H. (1998). Putting the enterprise into the enterprise system. Harvard Business Review, 76(4), 121-131.

Davenport, T. H. (2000). Mission critical: Realizing the promise of enterprise systems. Boston, MA: Harvard Business School Press.

Davenport, T. H., Harris, J. G., \& Cantrell, S. (2004). Enterprise systems and ongoing process change. Business Process Management Journal, 10(1), 16-26. doi:10.1108/14637150410518301

DeSanctis, G., \& Gallupe, R. B. (1987). A foundation for the study of group decision support systems. Management Science, 33(5), 589-609. doi:10.1287/ mnsc.33.5.589
DeSanctis, G., Poole, M. S., \& Zigurs, I. (2008). The Minnesota GDSS Research Project: Group support systems, group processes, and outcomes. Journal of the Association for Information Systems, 9(10), 6 .

Douma, A., Schuur, P., \& Jagerman, R. (2010). Degrees of terminal cooperativeness and the efficiency of the barge handling process. Expert Systems with Applications: an International Journal, 38(4), 3580-3589. doi:10.1016/j.eswa.2010.08.147

Douma, A. M. (2008). Aligning the operations of barges and terminals through distributed planning. Enschede, The Netherlands: Universiteit Twente Enschede.

Eckartz, S., Katsma, C., \& Daneva, M. (2010). The inter-organizational business case in ES implementations: Exploring the impact of coordination structures and their properties. Paper presented at the Conference on Enterprise Information Systems, Viana do Castelo, Portugal.

Fenton, N., \& Pfleeger, S. L. (1996). Software metrics: a rigorous and practical approach. Boston, MA: PWS.

Ferrario, L. I., \& Montagna, J. M. (2004). A framework for evaluating difficulties in ERP implementation. Paper presented at the International Conference on Enterprise Information Systems - Databases and Information Systems Integration, Porto, Portugal.

Heide, J. B. (1994). Interorganizational governance in marketing channels. Journal of Marketing, 58, 71-85. doi:10.2307/1252252

Hofstede, G. (1983). Cultural dimensions for project management. International Journal of Project Management, 1(1), 41-48. doi:10.1016/02637863(83)90038-8

Hong, I. B. (2002). Anew framework for interorganizational systems based on the linkage of participants' roles. Information \& Management, 39(4), 261-270. doi:10.1016/S0378-7206(01)00095-7

Jaffee, D. (2001). Organization theory: Tension and change. New York, NY: McGraw-Hill.

Kendra, K., \& Taplin, L. J. (2004). Project success: A cultural. framework. Project Management Journal, $35,30-45$.

Kishore, R., Zhang, H., \& Rameshc, R. (2004). Enterprise integration using the agent paradigm: foundations of multi-agent-based integrative business information systems. Decision Support Systems, 42(1), 48-78. doi:10.1016/j.dss.2004.09.011 
Klein, R., Kupsch, F., \& Scheer,A. (2004). Modellierung interorganisationaler Prozesse mit Ereignisgesteuerten Prozessketten. In Scheer, A. (Ed.), Research report of the Institute for Information Systems (No. 178). Saarbruecken, Germany: Saarland University.

Kumar, K., \& van Hillegersberg, J. (2000). Enterprise resource planning: Introduction. Communications of the ACM, 43(4), 22-26. doi:10.1145/332051.332063

Lam, S. S., \& Schaubroeck, J. (2000). Improving group decisions by better pooling information: A comparative advantage of group decision support systems. The Journal of Applied Psychology, 85(4), 565-573. doi:10.1037/0021-9010.85.4.565

Malone, T. W., \& Crowston, K. (1994). The interdisciplinary study of coordination. ACM Computing Surveys, 26(1),87-119. doi:10.1145/174666.174668

McCall, J. A., Richards, P. K., \& Walters, G. F. (1977). Factors in software quality (Tech. Rep. No. ADA049014). Sunnyvale, CA: General Electric.

Oliver, C. (1990). Determinants of interorganizational relationships: Integration and future directions. Academy of Management Review, 15(2), 241-265.

Park, S. H. (1996). Managing an interorganizational network: A framework of the institutional mechanism for network control. Organization Studies, 17(5), 795-824. doi:10.1177/017084069601700505

Powel, W. W. (1990). Neither market nor hierarchy: Network forms of organization. Research in Organizational Behavior, 12, 295-336.

Provan, K. G., \& Kenis, P. (2007). Modes of network governance: Structure, management, and effectiveness. Journal of Public Administration Research, 18, 229-252. doi:10.1093/jopart/mum015

Remenyi, D. (1999). IT investment-making a business case. Oxford, UK: Butterworth-Heinemann.

Rowley, J., \& Slack, F. (2007). Information kiosks: a taxonomy. The Journal of Documentation, 63(6), 879-897. doi:10.1108/00220410710836402

Schein, E. H. (2004). Organizational culture and leadership. New York, NY: John Wiley \& Sons.

Schmidt, M. J. (2003a). The IT business case: Keys to accuracy and credibility. Boston, MA: Solution Matrix.
Schmidt, M. J. (2003b). What's a business case? And other frequently asked questions. Boston, MA: Solution Matrix.

Schubert, P., \& William, S. (2009). An extended framework for comparing expectations and realized benefits of enterprise systems implementations. Paper presented at the Fifteenth Americas Conference on Information Systems, San Francisco, CA.

Schulz, K., \& Orlowska, M. (2004). Facilitating cross-organisational workflows with a workflow view approach. Data \& Knowledge Engineering, 51(1), 109-147. doi:10.1016/j.datak.2004.03.008

Shang, S., \& Seddon, P. B. (2002). Assessing and managing the benefits of enterprise systems: the business manager's perspective. Information Systems Journal, 12(4), 271-299. doi:10.1046/j.13652575.2002.00132.x

Thompson, L. (1990). Negotiation behavior and outcomes: Empirical evidence and theoretical issues. Psychological Bulletin, 108(3), 515-532. doi:10.1037/0033-2909.108.3.515

Thorelli, H. B. (1986). Networks: Between markets and hierarchies. Strategic Management Journal, 7(1), 37-51. doi:10.1002/smj.4250070105

van Alstyne, M. W. (1997). The state of network organization: A survey in three frameworks. Journal of Organizational Computing, 7(3). doi:10.1080/10 919392.1997.9681069

Ward, J., \& Daniel, E. (2006). Benefits management: Delivering value from IS\& IT investments. New York, NY: John Wiley \& Sons.

Ward, J., Daniel, E., \& Peppard, J. (2008). Building better business cases for IT investments. MIS Quarterly Executive, 7(1), 1-15.

Webster, J., \& Watson, R. T. (2002). Analyzing the past to prepare for the future: Writing a literature review. Management Information Systems Quarterly, 26(2), xiii-xxiii.

Xu, L., \& Beamon, B. M. (2006). Supply chain coordination and cooperation mechanisms: an attribute-based approach. Journal of Supply Chain Management, 42(1), 4-12. doi:10.1111/j.1745493X.2006.04201002.x 
Silja Eckartz, originally from Germany, is a PhD-Researcher in the Information Systems group at the University of Twente, the Netherlands. Her research interests are cost and benefit estimation for inter-organizational IS implementations and business case analysis. She is in her last year of her four-year PhD trajectory and expected to finish her dissertation in 2012. Prior to joining her current research group in Twente, she worked at Maastricht University, the Netherlands, from where she also received her Bachelor and Master in International Business. She is a member of the Beta Gamma Sigma Honor Society.

Christiaan Katsma is an assistant professor in the department of Information Systems and Change Management. His research includes implementation of ICT and organizational change, and recently the adoption and design of hedonic information systems with an emphasis on digital music services. In his research he investigates new implementation methodologies in relation to organizational learning, human behavior and intervention mechanisms. Secondly he investigates functionalities and business models of online music services. Prior to his position at the university he worked in Germany and Japan in the chemical and ICT industry. In this period he was involved in research and development and as an implementation consultant. Christiaan is also involved in various post academic curricula for professionals. He has authored and coauthored various journal and conference papers and is one of the founding fathers of the Dutch implementation interest group.

Maya Daneva is an assistant professor with the Information Systems Group at the University of Twente, the Netherlands. Her research is focused on business-IT alignment, maturity models, requirements engineering, cost estimation of large systems, and the application of qualitative research methods. Maya also serves as a liaison to the Dutch IT industry. Prior to this, Maya spent 9 years as a business process analyst in the Architecture Group of TELUS, Canada's second largest telecommunication company in Toronto, where she consulted on ERP requirement engineering processes and their evaluation as well as on ERP-supported coordination models and business process design. Maya authored more than 70 research and experience papers. 